\title{
Article
}

\section{Research on an SVM Coupling Algorithm of Image recognition}

\author{
Ai-Min Yang ${ }^{1}$, Yang Han ${ }^{1}$, Jin-Ze Li ${ }^{2}$, Yu-hang Pan ${ }^{1}$, Lei-Jie Shen ${ }^{1}$, Yan Long ${ }^{1}$ and Yu-Zhu \\ Zhang ${ }^{3, *}$ \\ 1 Hebei Key Laboratory of Data Science and Applications, North China University of Science and \\ Technology; aimin_heut@163.com \\ 2 College of Grassland and Environment Sciences, Xinjiang Agricultural University; LJZ_zky@163.com \\ 3 Ministry of Education, Key Laboratory with Modern Metallurgical Technology, North China University of \\ Science and Technology; zyz@ncst.edu.cn \\ * Correspondence: zyz@ncst.edu.cn; Tel.: +1-863-316-8516 \\ + Current address: North China University of Science and Technology, Tangshan, 063000,China \\ $\ddagger$ These authors contributed equally to this work.
}

\begin{abstract}
The key links of face recognition are digital image preprocessing, facial feature extraction and pattern recognition, this article aimed at the current problem of slow speed and low recognition accuracy of face recognition, from the above three key links, on the basic of analyzing the therories of Fractional Differential Masks Operator (FDMO), Principal Component Analysis (PCA) and Support Vector Machine (SVM), design a kind of FDMO+PVA+SVM coupling algorithm that applies to face recognition to improve the speed and accuracy of it. To realize FDMO+PCA+SVM coupling algorithm, first, we should apply FDMO to face image processing binary marginalization, the purpose is getting face contour; Then, we apply PCA to get the feature of face image which is disposed by binary marainalization. At last, we can apply One-Against All of the SVM classifier and LibSVM software package to realize face recognition. Beside, the article with nine different coupling algorithm design four groups of experimental reaults on the ORL face database verified by comparative analysic FDMO+PCA+SVM coupling algorithm in the superiority of face recognition accuracy and speed.
\end{abstract}

Keywords: Face Recognition; Intelligent Coupling Algorithm; Robustnes; Accuracy; Speed

\section{Introduction}

Interest in digital image processing methods stems from two principal application areas : improvement of pictorial imformation for human interpretation; and processing of image data for storage, transimission, and representation for autonomous machine perception. Among them, face recognition is an important application domain of digital image processing [1]. At present, many universities and research institutions around the world, the research institutions of MIT,CMU,Yale University,Tsinghua university, Chinese academy of sciences, Japan and Hong Kong have a special face recognition research group,face recognition technology is still researched by the scholars around the world, and constantly propose new theoretical approaches.Among them, Maria De Marsico, etc,came up with a kind of method which is based on Anti-partition iterated function systems's kang shelter and changes in the expression to face recognition, the method is based on strong robustness;Harin Sellahewa, etc [2](2010)came up with a proposed adaptive face recognition method which is based on image quality to overcome the influence of light conditions,Gu-Min Jeong came up with a new kind of shadow compensation method which is based on Fourier analysis to deals with the influence of illumination change. The scholars in the above did the study of the Human face image recognition, and they focused on the image preprocessing stage. After researching Digital image preprocessing [3], the application of fractional differential theory in image enhancement, edge detection and advance are more and more used in daily life. Also they achieved considerable results. Fuyuan Hu [4] (2013) proposed an adaptive fractional order differential load bilateral filtering 
algorithm, and formulated the fractional order differential mask and the algorithm of image texture enhancement; Mathieu B, et al [5] (2015) found that the common integer order differential edge detection operator can not keep the details of the image texture. He replaced the first derivative with 0-1 Order Fractional Differential and constructed three new operators for image edge detection. This study used non integer order differential G-L definition and combine with Parabolic edge detection method [6] is proposed for edge extraction of fractional differential mask operator, in order to lay a basis on face digital image preprocessing and image feature extraction. Support Vector Machine (SVM) [7] is developed in recent years in the field of statistical learning theory based on a new pattern recognition method.It can be used to solve the problem of small sample, nonlinear and high dimensional pattern recognition, show many unique advantages, and applied to the function fitting to other machine learning problems [8] .At present, SVM has become a hot research topic in the field of machine learning. It has been widely used in trend analysis, remote sensing image classification and pattern recognition, and so on.Including: the Zhi-feng Li,etc [9] (2007) using support vector machine (SVM) enhancement method based on Bayesian face recognition performance.Even though the method required less training samples, but the accuracy is not ideal;Shu Shuang Bao, etc [10] (2011) using a metric component analysis feature extraction and support vector machine to realize the multi class classification method for face recognition;Yang Yongqiang [11] (2015) proposed a new way of combining a discrete cosine transform with support vector machine (SVM) method for face recognition, reduce the time of face recognition, and improve the efficiency of identification.On the basis of studying a large number of documents, the writer prefers to regard the support vector machine as the classifier, which is used for face recognition. Yang Yongqiang proposed a new way of combining a discrete cosine transform with support vector machine (SVM) method for face recognition, reduce the time of face recognition, and improve the efficiency of identification.On the basis of studying a large number of documents, the writer prefers to regard the support vector machine as the classifier, which is used for face recognition.

\section{Theoretical Basis}

\subsection{Fractional Differential Masks Operator}

The fractional differential theory is applied to the digital image edge extraction and strengthen the high if the edge and texture information signal has a significant effect. Therefore, fractional differential for edge detection can effectively extract with high signal to noise ratio of the image edge and texture information [12]. In this study, based on the definition of non integer order differential G-L and the method of parabolic edge detection, this paper proposes a fractional differential mask operator for digital image edge detection based on [13]. G-L fractional order differential expression see formula (1), among them: $\alpha \in(0,1), h$ expresses as differential step length $(h=1)$, expresses Gamma function, $t$ and $a$ respectively expresses the upper and lower bounds of the fractional order differential.

$$
{ }_{a}^{G} D_{t}^{\alpha} f(t)=\lim _{h \rightarrow 0} \frac{1}{h^{\alpha}} \sum_{m=0}^{\left[\frac{t-a}{h}\right]}(-1)^{m} \times \frac{m ! \Gamma(\alpha-m+1)}{\Gamma(\alpha+1)} f(t-m h)
$$

From formula (1), differential expression (2) of the $\alpha$ order differential of a one-dimensional signal $f(t)$ can be obtained:

$$
\frac{d t^{\alpha}}{d^{\alpha} f(t)} \approx f(t)+(-\alpha) f(t-1)+\ldots+\frac{m ! \Gamma(-\alpha+m+1)}{\Gamma(-\alpha+1)} f(t-m)
$$

A one dimensional edge detection operator is constructed:

$$
\left\{\begin{array}{c}
\left(+a_{m}, \ldots,+a_{1},+a_{0},-a_{0},-a_{1}, \ldots-a_{m}\right) \\
a_{m}=(-1)^{m}\left(\begin{array}{c}
\alpha \\
m
\end{array}\right)=(-1)^{m} \frac{m !}{\alpha(\alpha-1) \ldots(\alpha-m+1)}
\end{array}\right.
$$


In order to construct a fractional order differential edge detection operator mask with rotation invariance for two-dimensional digital image $f(x, y)$, using $0, \pi / 8, \pi / 4,3 \pi / 8, \pi / 2,5 \pi / 8,3 \pi / 4,7 \pi / 8$ and the edge detection operator in 16 directions, at the same time using the $5 \times 5$ size of the window $w$, the mask is shown in Figure 1-a, the output of the edge image $g(x, y)$ can be expressed as a discrete convolution (4) as shown in the form:

$$
g(x, y)=\sum_{s=-2}^{2} \sum_{t=-2}^{2} f(x+s, y+t) w(s, t)
$$

When testing image edge, the threshold is selected according to the characteristics of the image. By design of the mask can be seen, the mask coefficient and 0 , so the convolution of the pixels in the smooth regions response to 0 , and the high-frequency edge and texture details but very sensitive, can be keen to capture the texture changes, rich texture edge can be extracted, at the same time the mask mode does not significantly increase the computational complexity. In this study, the image gray level is divided into 256, respectively using the Sobel operator and the designed fractional order differential edge detection operator $(\alpha=0)$ to MATLAB image processing with the edge of the image Lena image to extract, the results are shown in Figure 1-b and Figure 1-c. From the vision, Mask operator detection effect obviously is better, fractional differential edge detection operator is superior to other methods in extracting texture details on.

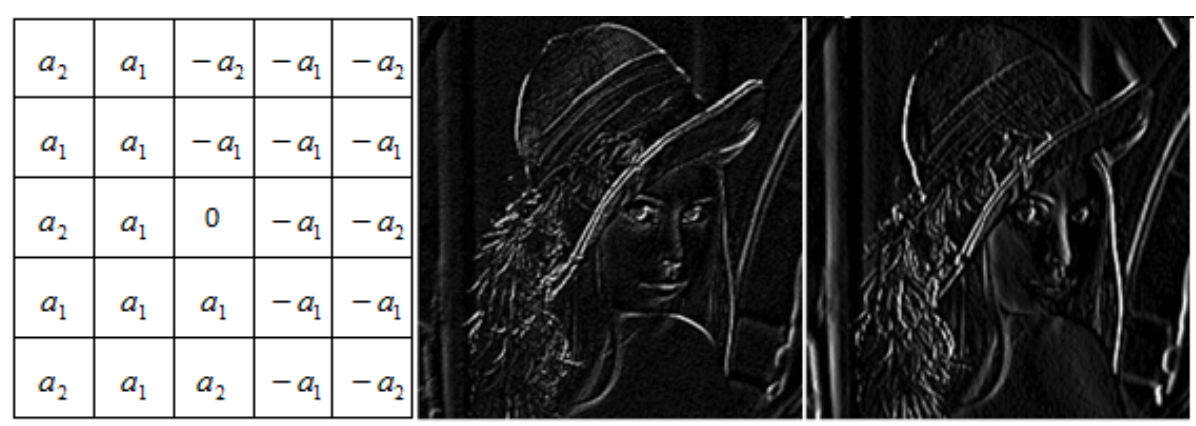

Figure 1. (1-a) Detection template; (1-b) Sobel operator detection effect; (1-c)FDMO operator detection effect

\subsection{Facial Feature Extraction Based on PCA}

PCA is principal component analysis, the method is an effective method to analyze the data in statistics, first proposed by Turk and Pentland [11], its basis is the Karhunen-Loeve transform (that is, K-L transform). Using K-L transform, a low dimensional subspace is used to describe the face image, and it can be used to identify the important information. Using the training sample to set as a general walk matrix to generate the matrix of feature vectors, that is:

$$
S_{t}=\frac{1}{N} \sum_{n=1}^{N}\left(\Gamma_{n}-\Psi\right)\left(\Gamma_{n}-\Psi\right)^{T}
$$

Among function (5), $N$ is total number of training samples, $\Psi$ is the average image vector of the training sample set, $\Gamma_{n}$ is the $n$th image vector of training samples. A set of orthogonal feature vectors for the generation of the matrix is obtained, that is "eigenface". Anyone's face image can have sub space projection according to the feature of "eigenface" and obtain a set of coordinate coefficient, tant is K-L decomposition coefficient. The coefficients indicating position of the image in the feature space, which can be used as the basis for face recognition. 


\subsection{Support Vector Machine (SVM)}

If all vectors in the training set could be correctly divided by a hyperplane and the spacing between heterogeneous being nearest to the hyperplane is maximum, the hyperplane shall be deemed as optimal hyperplane [14]. Heterogeneous vectors being nearest to the hyperplane are called as support vectors (SV), a group of support vectors can uniquely identify a hyperplane. SVM evolves from the optimal classification plane under linearly separable conditions, which basic principle can be shown in Figure 2. The solid points and hollow points represent two kinds of samples. H is the classification hyperplane between them, $\mathrm{H} 1$ and $\mathrm{H} 2$ are the samples being nearest to classification hyperplane, and parallel to classification hyperplane. The distance between them is called as margin.

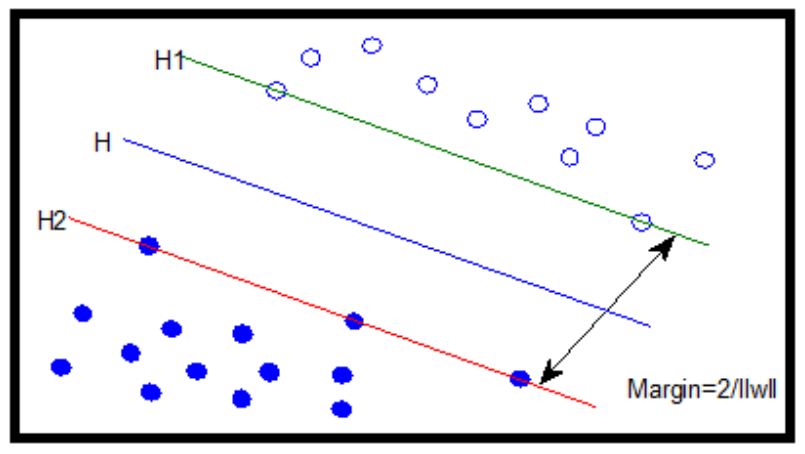

Figure 2. Basic principles of SVM

Assuming the linearly separable set of samples is $\left(x_{i}, y_{i}\right), i=1,2, \ldots, n ; x \in R_{d}, u \in+1,-1$ is class label; in d-dimensional space, the general form of linear discriminant function is $g(x)=w \cdot x+b$, then the classification hyperplane equation is . The discriminant function is normalized, and thus all two types of samples meet the condition $|g(x)| \geq 1$. Even if $|g(x)|=1$ for the sample being nearest to classification hyperplane, this classification margin is equivalent to $\frac{2}{\|\omega\|}$. Therefore, the margin maximum is equivalent to $\|\omega\|^{2}$ minimum. If the classification hyperplane is required to correctly classify all the samples, the equation (6) shall be satisfied:

$$
y_{i}\left[\left(\mathbf{w} \cdot \mathbf{x}_{i}\right)\right]-1 \geq 0, i=1,2, \ldots, n
$$

Therefore, if satisfying the equation (6) and enabling $\|\omega\|^{2}$ to be minimized, such the hyperplane shall be the optimal classification hyperplane. If the training samples are nearest to the classification hyperplane, and parallel to hyperplanes $\mathrm{H} 1$ and $\mathrm{H} 2$ in the optimal classification hyperplane [15], they should be able to enable the equation (6) to be equal, and they are called SV. In linear separable case, the optimal hyperplane problem under structural risk minimization is considered as an optimization problem where objective function is to minimize $\Phi(\omega)=\frac{1}{2}\|\omega\|^{2}$. Therefore, it is available to define as Lagrange function as shown in equation (7) below, in the equation $a_{i} \geq 0$ is Lagrange coefficient corresponding to each sample.

$$
L(\omega, b, a)=\frac{1}{2}\|\omega\|^{2}-\sum_{i=1}^{n} a_{i} y_{i}\left(\mathbf{w} \cdot \mathbf{x}_{i}+b_{i}\right)+\sum_{i=1}^{n} a_{i}
$$

When the solving equation (7) is minimized, variational function seeks partial derivative to and , and make them to be equal to 0 ; then it is available to transfer optimal classification hyperplane problem into simple dual problem, namely solving $\alpha_{i}$ in case of the maximum value of the function as shown in equation (3) under the constraint conditions $\sum_{i=1}^{n} y_{i} \alpha_{i}=1$ and $\alpha_{i} \geq 0$. If $\alpha_{i}$ is the optimal solution, $\omega^{*}=\sum_{i=1}^{w} \alpha_{i}^{*} y_{i} x_{i}$ will be the weight coefficient vector of optimal classification hyperplane, namely 
the weight coefficient vector of optimal classification hyperplane is a linear combination of support vectors in training samples.

$$
Q(\alpha)=\sum_{i=1}^{n} \alpha_{i}-\frac{1}{2} \sum_{i, j=1}^{n} \alpha_{i} \alpha_{j} y_{i} y_{j}\left(\mathbf{x}_{i} \cdot \mathbf{x}_{j}\right)
$$

According to the functional theory, as long as a kernel function $K\left(x_{i}, x_{j}\right)$ satisfies Mercer condition, it will correspond to inner product in a transformation space [15-18]. Mercer condition specifies: any symmetric function $K\left(x_{i}, x_{j}\right)$ is a necessary and sufficient condition for inner product operation in a feature space; as for any $\varphi(x) \neq 0$ and $\int \varphi^{2}(x) d x<\infty$, the equation (9) is established as follows:

$$
\iint K\left(x, x^{\prime}\right) \varphi\left(x^{\prime}\right) d x d x^{\prime} \geq 0
$$

SVM classification function is a set of linear combinations of nonlinear functions with support vectors as parameters. The SVM classification function only related to the number of SVM, and has nothing to do with the space dimensions. Therefore, even if the dimension of the space changes increase a lot, but the optimal classification function does not increase too much time complexity. The working principle of dealing with nonlinear SVM as shown in Figure 3:

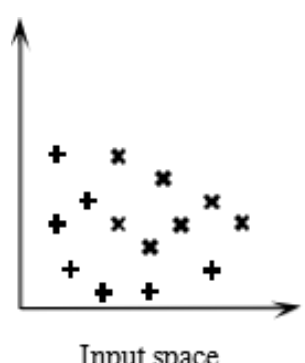

Input space
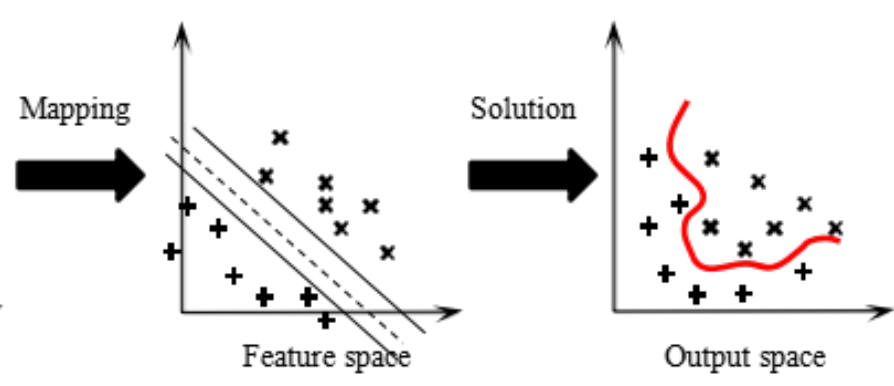

Figure 3. The solution of SVM to nonlinear problems

By as shown in type (10) function expressions of support vector machine, shows that the SVM through the inner integral kernel function the original low dimensional space transform to the new high dimension space, and classification of SVM discriminant relate only to be recognition of the sample and the training samples of the support vector.

$$
f(x)=\operatorname{sgn}\left(w^{*} \cdot x+b\right)=\operatorname{sgn}\left(\sum_{i=1}^{n} \alpha_{i}^{*} y_{i} K\left(x_{i} \cdot x\right)+b^{*}\right)
$$

Using the kernel function included [16]: linear kernel function $K\left(x_{i}, x_{j}\right)=x_{i}^{T} x_{j}$, Polynomial inner product function $K\left(x_{i}, x_{j}\right)=\gamma x_{i}^{T} x_{j}+r^{d}, \gamma>0$, radial basis kernel function $K\left(x_{i}, x_{j}\right)=$ $e^{-\gamma\left\|x_{i}-x_{j}\right\|^{2}}, \gamma>0$, sigmoid function $K\left(x_{i}, x_{j}\right)=\tanh \left(\gamma x_{i}^{T} x_{j}+r\right)$. In this paper, the application of kernel function is radial basis kernel function.

\section{Coupling Algorithm Design}

This study uses a fractional differential mask operator norm, the face digital image edge detection, extraction of binary edge image and realize the digital image preprocessing, the influences to abate illumination and expression variations of facial feature. Two in the face value of the edge extraction based on the principal component analysis method for face image feature extraction, realize the evaluation of face essence in order to use less index. Finally, we use the support vector machine model of the sample interpretation, the realization of face recognition based on digital image. The 
algorithm process of binary edge manage of face feature that based on the measure of FMDO. As shown in Figure 4.

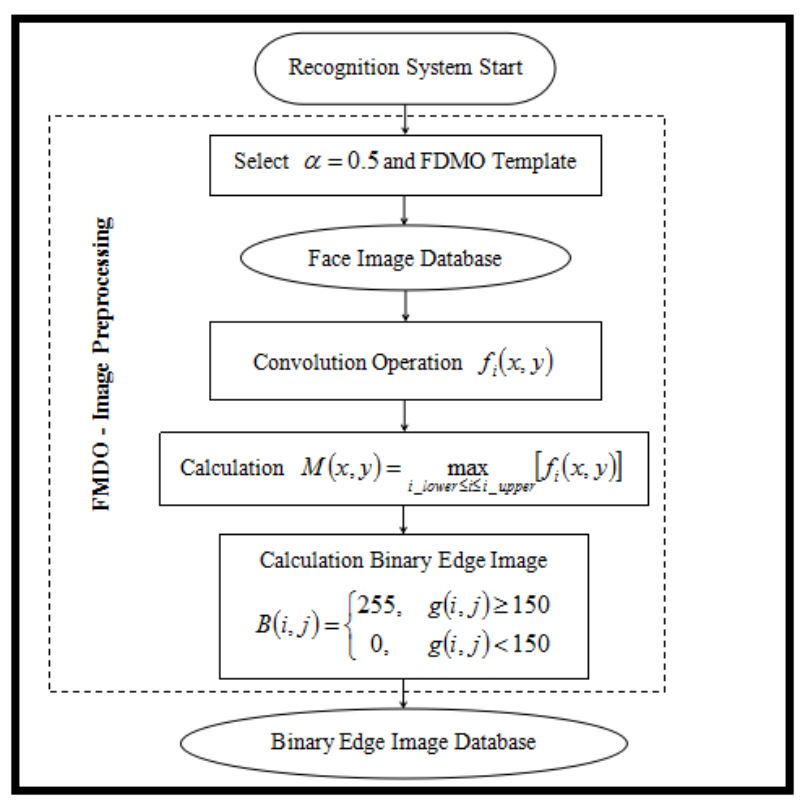

Figure 4. The algorithm process of binary edge manage of face feature that based on the measure of FMDO

Assuming that there are $c$ categories, each category to take the same number of training samples, a total of training samples. Pixel gray value of each training sample face $I(x, y)$ is according to $(x)$ first and $(y)$ latter to make up a vector $\Gamma_{n}(M=x \cdot y)$ of a length of $M$, which can be thought a data point in $M$-dimensional space of original image. The sample set consisting of $N$ vectors is $\Gamma_{1}, \Gamma_{2}, \ldots, \Gamma_{N}$. "Characteristic face" calculation process, as shown in Figure 5: 


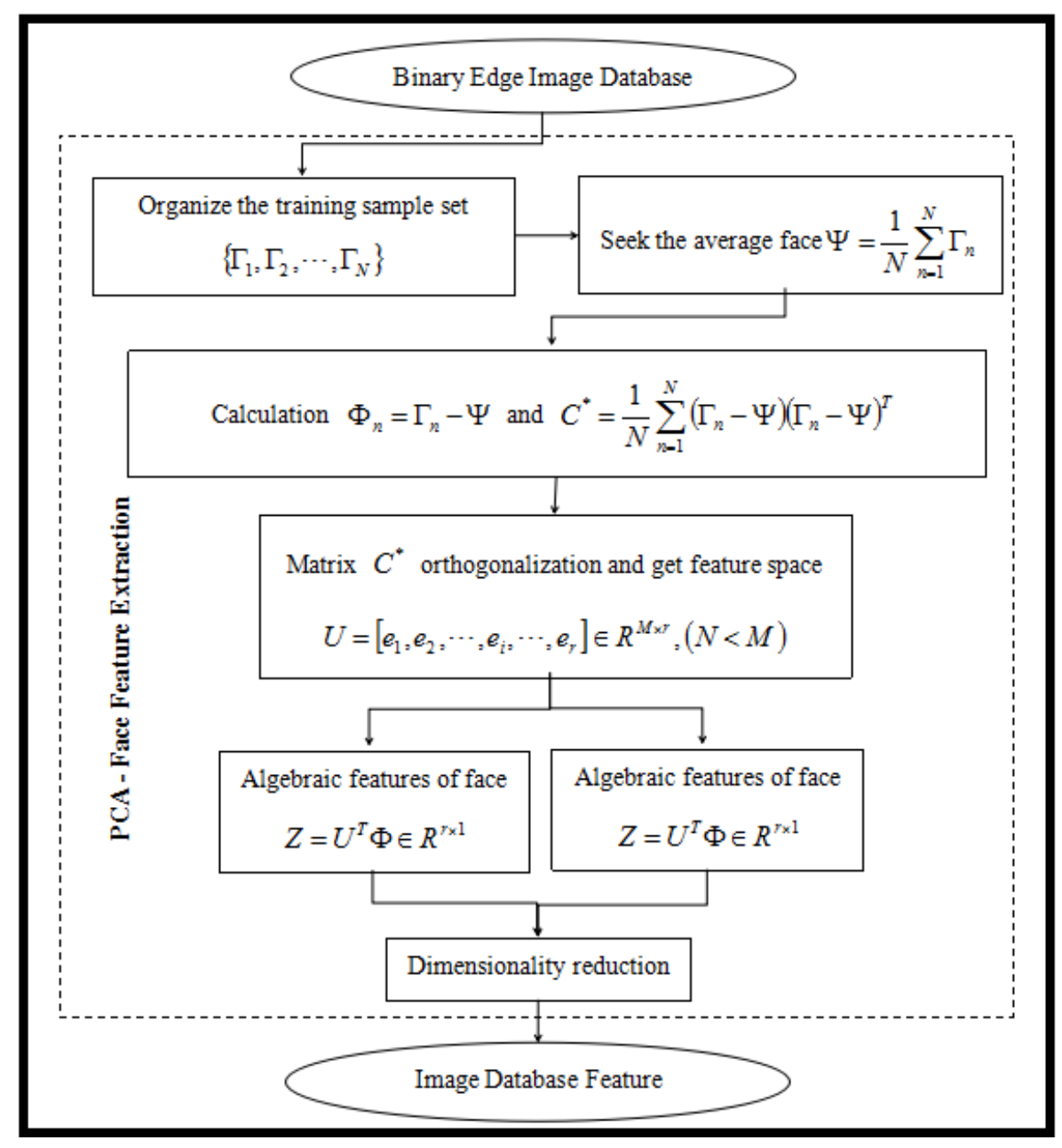

Figure 5. Calculating Process of "Eigenfaces"

SVM in essence is two classifiers, if use two types of classifier for classifying multiple kind of pattern recognition problems of classifier, usually with One Against All (OAA), One Against One $(\mathrm{OAO})$ and Multi - class Objective Functions provides (MCOF). Where: OAA method is tocategories, each to one of the classes seen as positive samples, the rest of the class as negative samples to classify, a total need $\frac{c(c-1)}{2}$ of classifiers. At the time of classification, take a sample of the given classifier for classification, each classifier will give a class label, the category label statistics, category label class most category is to sample points belong to the category of the. At present, LibSVM and SMO algorithm is effective to solve the SVM . LibSVM is a Taiwan University zhi-ren Lin develop designed a simple and fast, efficient and easy to use pattern recognition and regression SVM software package, which involves less parameter adjustment, and provides cross-validation function. SMO is a fast and effective algorithm of support vector machine, which transforms the large convex programming problem into a lot of small convex programming problem, and can be trained a large data sets. The classification method used in this paper is the OAA method, application of LibSVM and SMO algorithm. The face recognition algorithm process that based on the coupling of FMDO+PCA+SVM , As shown in Figure 6. 


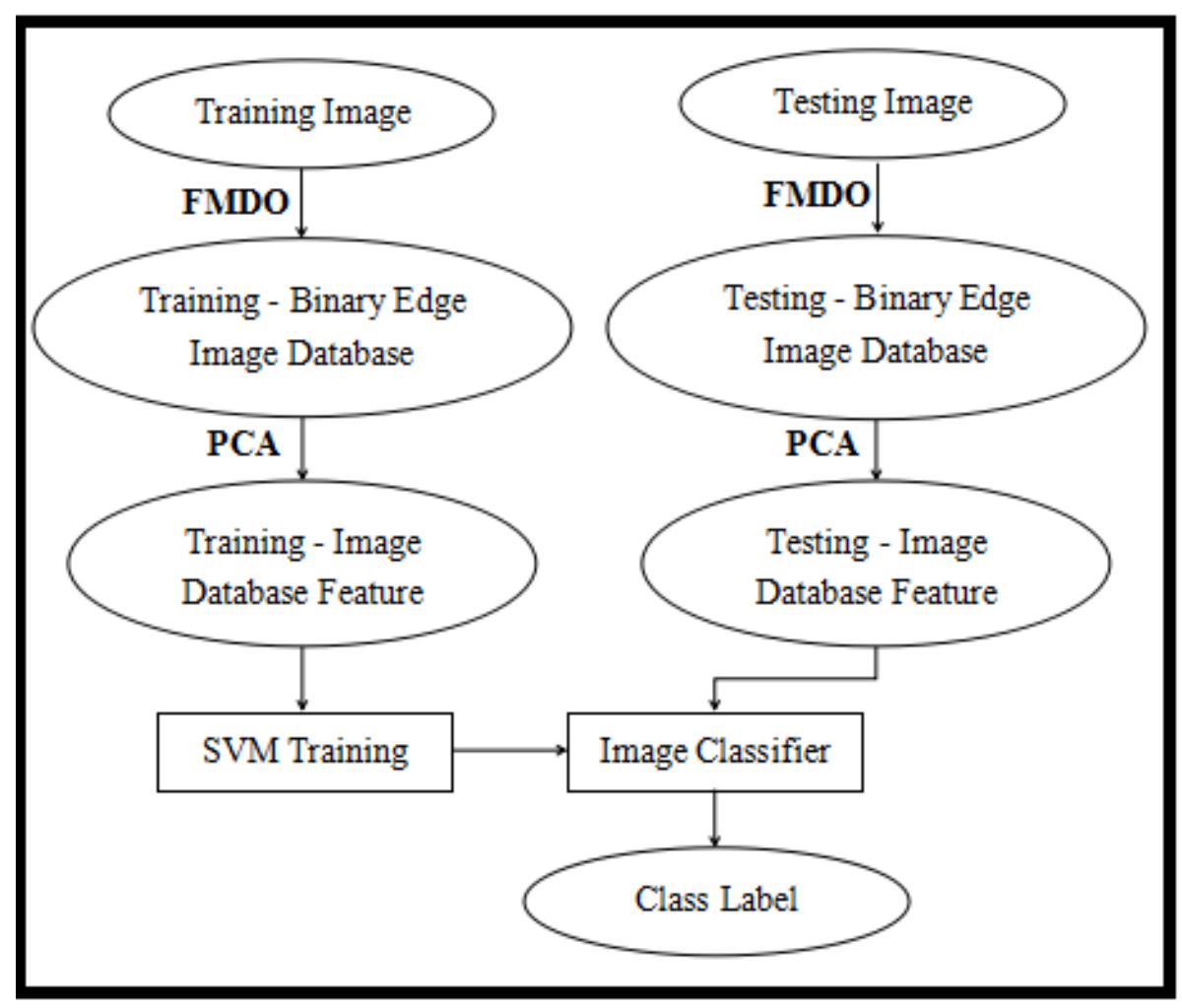

Figure 6. The face recognition algorithm process that based on the coupling of FMDO+PCA+SVM

\section{Experiment Design and Result Analysis}

\subsection{Experiment Design}

This experiment is carried out on the ORL face database,The ORL database is the University of Cambridge in the United Kingdom AT and T labs acquisition, contains 40 person, 10 images per person, per image to 256 grayscale, $92 \times 112$ pixels, in different time, different expressions, different illumination and obtained under different attitude can reaction with a different face image changes and differences.Experiments using ORL face database in each of the first 5 images, different people's total of 200 images, $20 \times 8$ face used to train, $20 \times 2$ face to identify. The SVM classifier in the experiment chooses the same design parameters and classification strategies, which include the penalty factor $C=1, \gamma=2$, and the classification strategy for the OAA method.The recognition algorithm is based on PCA+NN, SVM and PCA+SVM. The nine algorithms in Table 1 are designed to verify the superiority of FDMO+PCA+SVM algorithm. The experimental procedure is as follows: 1) Number of identification algorithm, the number of the table as shown in table 1.

Table 1. The table of recognition algorithm of number.

\begin{tabular}{cccccc}
\hline Number & Method & Number & Method & Number & Method \\
\hline 1 & PCA+NN & 4 & Sobel+PCA+NN & 7 & FDMO+PCA+NN \\
2 & SVM & 5 & Sobel+SVM & 8 & FDMO+SVM \\
3 & PCA+SVM & 6 & Sobel+PCA+SVM & 9 & FDMO+PCA+SVM \\
\hline
\end{tabular}

2)Number of the experimental categories, including Experiment-1 of the training sample is 60, the test sample is 140; Experiment-2 training samples are 100. The test sample are 100 Experiment-3; the training samples are 120, 280 test samples; training sample of Experiment- 4 are 200. The test 
sample are 200. 3)Four experiments were carried out using 9 kinds of recognition algorithms in Table 1 , then the recognition accuracy and recognition speed were recorded.

\subsection{Results and Analysis of Binary Edge Processing of Face Image}

The edge image has better robustness to illumination.In order to reduce the computation time, the fractional differential mask operator is used to extract the edge of the face image, and binarization image edge.Mask operator is shown in Figure 7,Among them: $\alpha=0.5$ the fractional order.The fractional order differential of the gray image $f$ convolution operation, the imagewill be enhanced,and select the gray value 150 as the threshold, and threshold value the two edge image $B(i, j)=\left\{\begin{array}{ll}255 & \text { if } g(i, j) \geq 150 \\ 0 & \text { if } g(i, j)<150\end{array}\right.$.

\begin{tabular}{|c|c|c|}
\hline$\frac{\alpha}{8 \alpha-8}$ & $\frac{\alpha}{8 \alpha-8}$ & $\frac{\alpha}{8 \alpha-8}$ \\
\hline$\frac{\alpha}{8 \alpha-8}$ & $\frac{8}{8-8 \alpha}$ & $\frac{\alpha}{8 \alpha-8}$ \\
\hline$\frac{\alpha}{8 \alpha-8}$ & $\frac{\alpha}{8 \alpha-8}$ & $\frac{\alpha}{8 \alpha-8}$ \\
\hline
\end{tabular}

Figure 7. Operator FDMO based on fractional differential
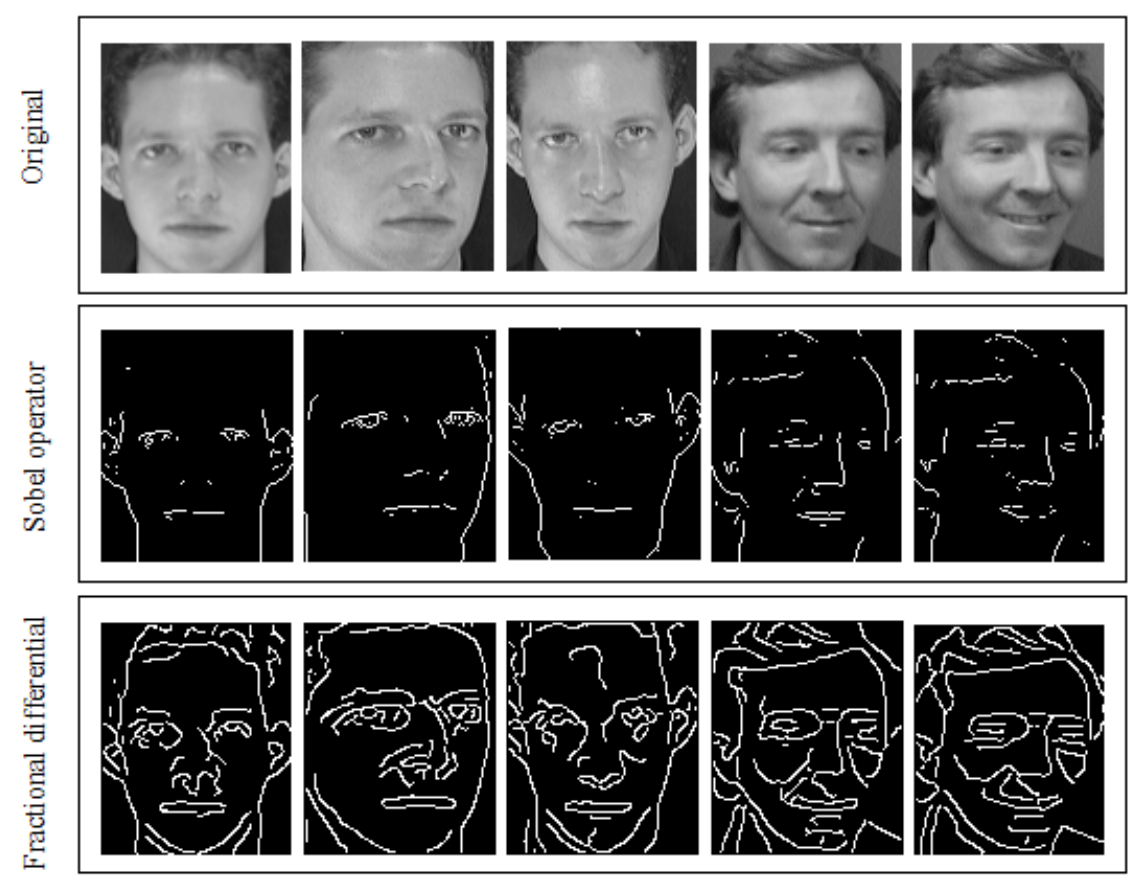

Figure 8. The partial face images and their binary edge images on ORL face database

As shown in Figure 8, the partial face image two value edge processing results. The results show that the two valued edge image based on fractional order differential extraction, the object contour 
is prominent, the edge details are clear, which can eliminate the influence of illumination and facial expression changes, and has strong robustness.

\subsection{Face Recognition Result Analysis}

Face recognition results are shown in table 2:

Table 2. The comparsion result of recognition accuracy and speed.

\begin{tabular}{ccccccccc}
\hline Method & Acuracy-1 & Speed-1 & Acuracy-2 & Speed-2 & Acuracy-3 & Speed-3 & Acuracy-4 & Speed-4 \\
\hline 1 & 90.00 & 1.235 & 92.00 & 0.984 & 80.36 & 2.906 & 88.00 & 2.484 \\
2 & 85.71 & 3.750 & 85.71 & 3.422 & 78.93 & 12.328 & 84.00 & 11.000 \\
3 & 94.29 & 11.860 & 92.00 & 6.814 & 81.10 & 57.334 & 85.00 & 44.093 \\
4 & 92.37 & 3.017 & 92.89 & 2.157 & 88.33 & 3.259 & 94.02 & 3.735 \\
5 & 90.25 & 3.825 & 89.23 & 2.474 & 90.95 & 5.373 & 91.21 & 4.911 \\
6 & 96.44 & 5.723 & 95.91 & 3.219 & 92.66 & 20.715 & 95.62 & 22.105 \\
7 & 93.75 & 2.530 & 94.01 & 2.033 & 90.91 & 2.715 & 92.12 & 3.153 \\
8 & 96.92 & 2.151 & 95.43 & 2.421 & 95.01 & 3.711 & 95.75 & 3.517 \\
9 & 99.82 & 2.985 & 99.21 & 3.105 & 98.92 & 4.426 & 99.02 & 6.092 \\
\hline
\end{tabular}

As shown in Figure 9, PCA+NN, SVM, PCA+SVM algorithm four times the results of the experiment:
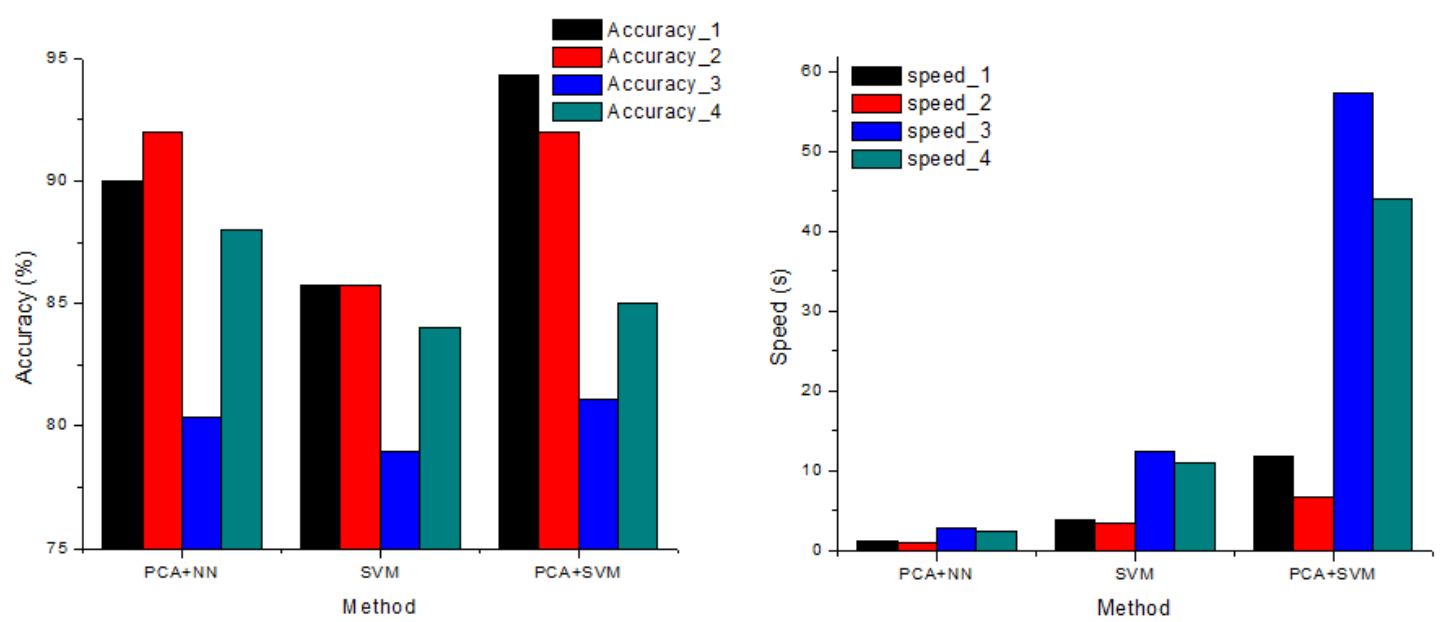

Figure 9. The comparison of four experimental results in PCA+NN, SVM, PCA+SVM

From table 2 and Figure 9 shows the characteristics show that, under the condition of less samples, PCA + SVM in the recognition accuracy was significantly better than the other two methods, and the recognition speed to slow to the other two, simple methods of SVM in the recognition accuracy than the other two methods at a disadvantage.In general, $P C A+N N$, as a classical face recognition algorithm, has its advantages in recognition rate and time.In general, PCA+NN, as a classical face recognition algorithm, has its advantages in recognition rate and time.As a new machine learning algorithm, the SVM method has better classification performance in small sample cases. Comparison of the Figure 10 and Figure 9 shows that, the first application of Sobel operator for binary image edge extraction and face recognition accuracy and speed have been improved to some extent, and the accuracy has been significantly improved, speed improved significantly.Comparison of Figure 8, Figure 10 and Figure 11, shows that FDMO algorithm has obvious superiority, the algorithm is not only suitable for small sample data, but also has good robustness in large sample. 


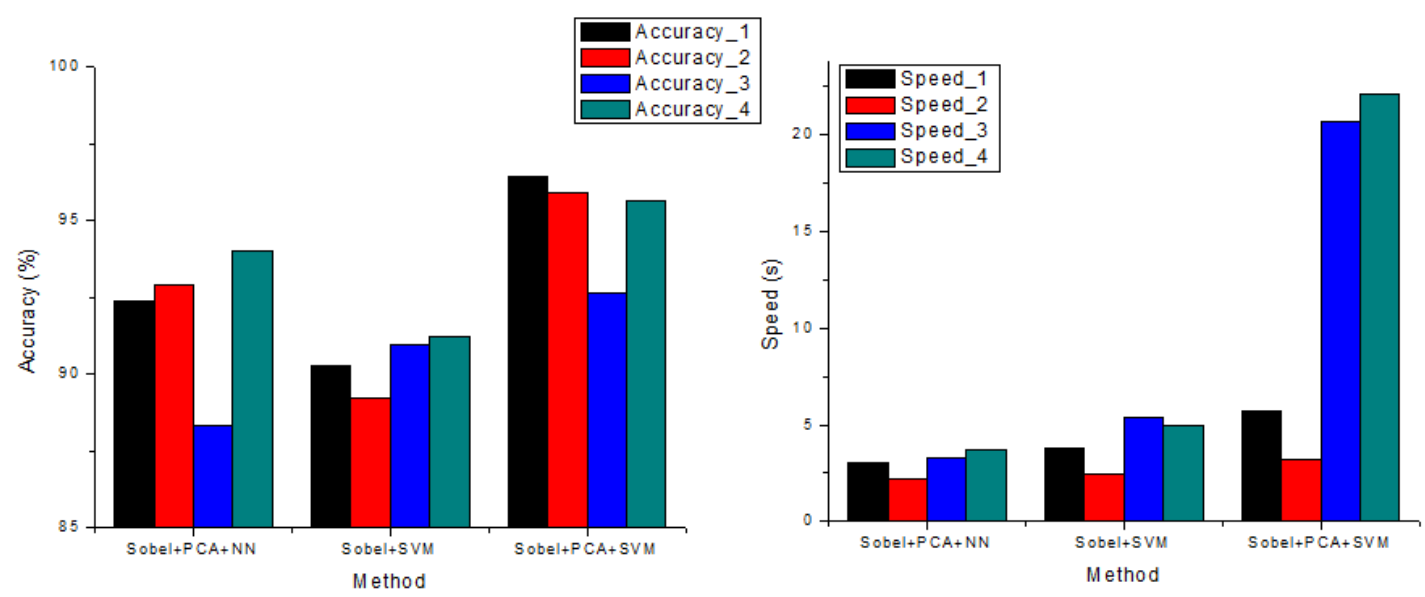

Figure 10. Comparison of four experimental results in Sobel+PCA+NN, Sobel+SVM, Sobel+PCA+SVM
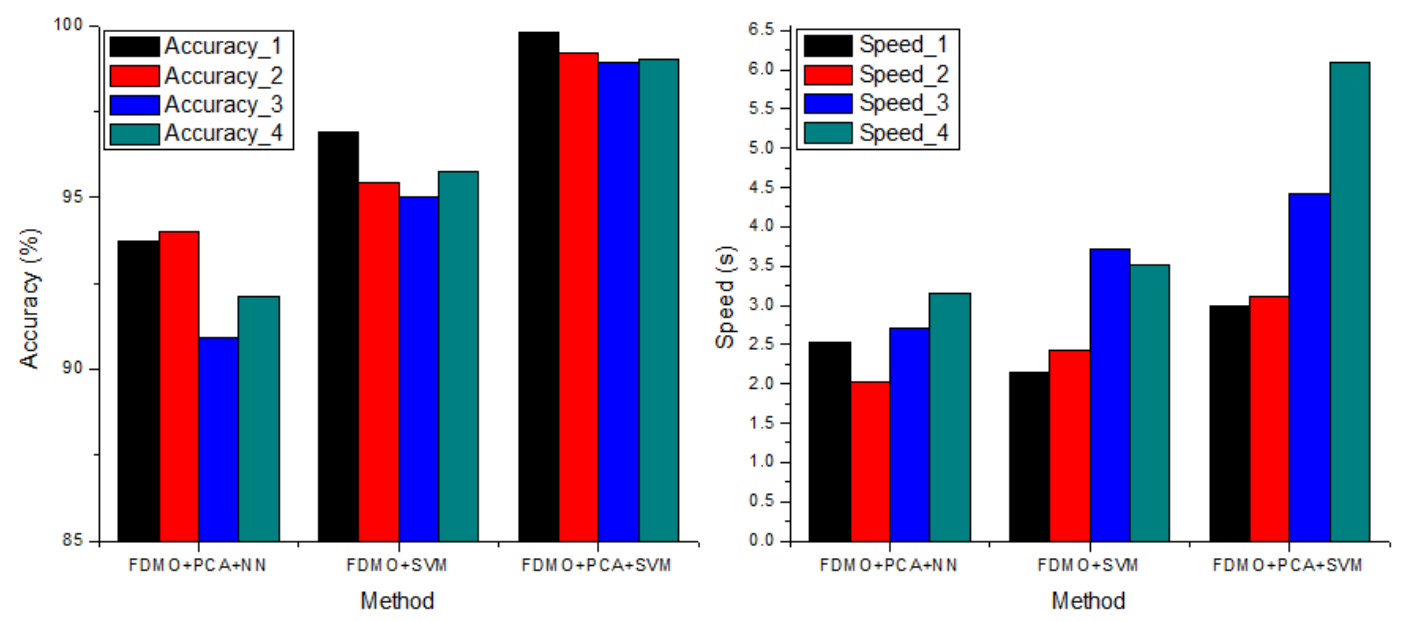

Figure 11. Comparison of four experimental results in FDMO+PCA+NN, FDMO+SVM, $\mathrm{FDMO}+\mathrm{PCA}+\mathrm{SVM}$

\section{Discussion}

The key to the performance of face recognition system lies in the selection and design of the recognition algorithm, and the evaluation index is the accuracy and speed of the algorithm .Therefore, the final goal of the algorithm is to improve the recognition accuracy and speed .Binary and marginalization processing of face image which is based on FDMO, not only to eliminate the influence of illumination and facial expression changes, but also laid the foundation for the fast extraction of facial features ;Facial feature extraction based on PCA, which can describe the human face image in a low dimensional subspace, and can be reserved for identification of important information, to ensure the recognition accuracy, but also reduce the input variables of the pattern recognition, provides a guarantee for improving pattern recognition rate; Using SVM algorithm for face recognition, can improve the recognition system for small sample data processing ability. The empirical results show that the FDMO+PCA+SVM algorithm has a significant advantage in terms of accuracy and speed. 
Acknowledgments: This work was supported by National Key Research and Development Program (no.2016YFB0601403) and the National Natural Science Foundation of Hebei Education Department (no. QN2016088).

Author Contributions: The idea of this study is proposed by Ai-Min Yang, Yang Han and Yu-Zhu Zhang. Jin-Ze Li, Yu-Hang Pan, Lei-Jie Shen and Yan Long for testing and data analysis. Ai-Min Yang and Yang Han to carry out the design and writing of the paper.

Conflicts of Interest: The authors declare no conflict of interest.

\section{References}

1. De Marsico M; Nappi M; Riccio D. FARO: FAce Recognition Against Occlusions and Expression Variations. IEEE Transactions on Systems Man and Cybernetics Part A Systems and Humans 2010, 40, 121-132.

2. Sellahewa H; Jassim S. Image-Quality-Based Adaptive Face Recognition. IEEE Transactions on Instrumentation and Measurement 2010, 59, 805-813.

3. Choi S I; Jeong G M . Shadow Compensation Using Fourier Analysis With Application to Face Recognition. IEEE Signal Processing Letters 2011, 18, 23-26.

4. Hu F Y; Si S H; Zhang Y N; Sun J Q. Multi-bilateral filtering algorithm based on adaptive fractional order differentiation. JOURAL OF IMAGE AND GRAPHICS 2013, 18, 1237-1246.

5. Mathieu B; Melchior P; Oustaloup A. Fractional differentiation for edge detection. Signal Processing 2003, 83, 2421-2432.

6. Wright J; Ma Y; Mairal J. Sparse Representation for Computer Vision and Pattern Recognition. Proceedings of the IEEE 2010, 98, 1031-1044.

7. Wright J; Ma Y; Mairal J. Sparse Representation for Computer Vision and Pattern Recognition. Encyclopedia of the Sciences of Learning 2010, 41, 3185-3185.

8. Jian G; Huang J; Qin Q. Face Detection Based on Hierarchical Support Vector Machines. Computer Engineering 2005, 31, 181-183.

9. Li Z F; Tang X O. Using Support vector machines to enhance the performance of bayesian face recognition . IEEE Transactions on Information Forensics and Security 2007, 02, 174-180.

10. Shu S B; Luo J R; Xu C D; Sun B X. A New Method of Face Recognition Base on Support Vector Machine. Computer simulation 2011, 28, 280-283.

11. Yang Y Q. Face Recognition Based on Fusing Discrete Cosine Transform and Support Vector Machine. Computer Applications and Software 2015, 32, 150-154.

12. Chen M J; Huang C H; Lee W L. A fast edge-oriented algorithm for image interpolation. Image and Vision Computing 2005, 23, 791-798.

13. Horn R A; Johnson C R. Matrix Analysis. A fast edge-oriented algorithm for image interpolation. University of Alberta.downloaded on December at from IEEE Xplore.restrictions Apply 1985, 1-17.

14. Rodrigues D; Freitas A C; Pereira L. Chemical composition of red, brown and green macroalgae from Buarcos bay in Central West Coast of Portugal. Food Chemistry 2015,183 197-207.

15. Shi Z; Han M. Support Vector Echo-State Machine for Chaotic Time-Series Prediction. IEEE Transactions on Neural Networks 2007,18 359-372.

16. Lin M; Zhang L; Jin R. Online kernel learning with nearly constant support vectors. Neurocomputing 2015,179 26-36.

(C) 2016 by the authors; licensee Preprints, Basel, Switzerland. This article is an open access article distributed under the terms and conditions of the Creative Commons Attribution (CC-BY) license (http://creativecommons.org/licenses/by/4.0/). 